\title{
A diversity-generating retroelement encoded by a globally ubiquitous Bacteroides phage
}

\author{
Sean Benler ${ }^{*^{*}}$ D, Ana Georgina Cobián-Güemes ${ }^{1}$, Katelyn McNair², Shr-Hau Hung ${ }^{1}$, Kyle Levi ${ }^{2}$, Rob Edwards ${ }^{2}$ \\ and Forest Rohwer ${ }^{1}$
}

\begin{abstract}
Background: Diversity-generating retroelements (DGRs) are genetic cassettes that selectively mutate target genes to produce hypervariable proteins. First characterized in Bordetella bacteriophage BPP-1, the DGR creates a hypervariable phage tail fiber that enables host tropism switching. Subsequent surveys for DGRs conclude that the majority identified to date are bacterial or archaeal in origin. This work examines bacteriophage and bacterial genomes for novel phageencoded DGRs.

Results: This survey discovered 92 DGRs that were only found in phages exhibiting a temperate lifestyle. The majority of phage-encoded DGRs were identified as prophages in bacterial hosts from the phyla Bacteroidetes, Proteobacteria, and Firmicutes. Sequence reads from these previously unidentified prophages were present in viral metagenomes (viromes), indicating these prophages can produce functional viruses. Five phages possessed hypervariable proteins with structural similarity to the tail fiber of BPP-1, whereas the functions of the remaining DGR target proteins were unknown. A novel temperate phage that harbors a DGR cassette targeting a protein of unknown function was induced from Bacteroides dorei. This phage, here named Bacteroides dorei Hankyphage, lysogenizes 13 different Bacteroides species and was present in $34 \%$ and $21 \%$ of whole-community metagenomes and human-associated viromes, respectively.

Conclusions: Here, the number of known DGR-containing phages is increased from four to 92. All of these phages exhibit a temperate lifestyle, including a cosmopolitan human-associated phage. Targeted hypervariation by temperate phages may be a ubiquitous mechanism underlying phage-bacteria interaction in the human microbiome.
\end{abstract}

Keywords: Bacteroides, Diversity-generating retroelements, Prophage, Viromes

\section{Background}

Phages encode genes selectively mutagenized by diversity-generating retroelements (DGRs) [1-3]. DGRs are genetic cassettes that introduce DNA sequence variation through a unique targeted mutagenesis mechanism [4]. The mechanism of DGR function is best understood in Bordetella phage BPP-1. BPP-1 possesses a 134 bp variable repeat $(\mathrm{VR})$ within the gene encoding the phage tail fiber. Downstream of VR is a second, invariant copy designated the template repeat (TR). Nearby the TR/VR pair are genes encoding an "accessory variability determinant" and a

\footnotetext{
* Correspondence: sbenler@gmail.com

'Department of Biology, San Diego State University, 5500 Campanile Drive, San Diego, CA 92182, USA

Full list of author information is available at the end of the article
}

reverse transcriptase (RT). These two proteins generate an error-prone cDNA from the TR, followed by stable incorporation of the mutagenized cDNA into the phage tail fiber gene $[4,5]$. This process, termed "mutagenic retrohoming," yields a VR that is distinct from the TR exclusively at adenine bases. As a result, the BPP-1 tail fiber that mediates adsorption to bacterial host receptors is hypervariable, enabling tropism switching on host Bordetella species $[4,6,7]$.

Since the initial discovery in BPP-1, the majority of DGRs identified to date are considered bacterial and archaeal in origin $[6,8-11]$. A recent survey found that $40 \%$ of DGRs are flanked by genes with sequence similarity to phages [12]. However, it is currently an open question whether these cassettes are encoded by functional prophages or inactive remnants. 
Here, a survey of phage genomes found that DGRs are only encoded by phages exhibiting a temperate lifestyle. Investigation of bacterial genomes found that DGRs previously considered to be bacterial in origin are encoded by functional prophages. These phage DGRs diversify proteins with unknown functions, though most possessed a C-type lectin domain. A single temperate phage, Hankyphage, was found to harbor a DGR cassette and lysogenize at least 13 different species of the genus Bacteroides. Hankyphage was present in whole-community metagenomes and viromes generated from microbial samples collected around the globe.

\section{Results}

\section{DGRs found in three temperate phages}

A comprehensive survey of phage genomes was conducted to identify novel phage-encoded DGRs. Searching publicly available genomes from isolated phages $(n=4203$, NCBI RefSeq and PhagesDB databases) identified six phages that possessed a reverse-transcriptase (RT). Three of these six possessed features characteristic of a DGR cassette: an adjacent variable repeat (VR)/template repeat (TR) pair containing $\geq 7$ adenine mismatches (Additional file 1). The paradigmatic DGR-harboring phage BPP-1 was identified, as well as two marine Vibrio phages. The VR of the BPP-1 DGR lay within the phage tail fiber gene, while the VRs of the two marine Vibrio phages were encoded within genes of unknown function. All three genes harbored a C-type lectin domain, consistent with previous structural analyses of DGR target proteins [13].

\section{DGRs encoded by novel temperate phages}

To more exhaustively search for DGRs in temperate phages, the survey was expanded to include temperate phages integrated into genomes as prophages. A database of 31,946 predicted prophage-containing regions generated by PhiSpy from 11,278 bacterial genomes was scanned for DGRs [14]. PhiSpy utilizes both sequence homology-dependent and independent approaches to predict regions harboring an integrated phage [15]. In total, 170 regions harbored a DGR cassette (Additional file 2). Previous surveys identified 25 of the 170 DGRs and assigned them as bacterial in origin, while 57 were reported as associated with a prophage or inactive phage remnant (Additional file 2) $[6,8,10-12,16]$. This analysis identified 74 novel DGRs and expands their observed association with temperate phages across diverse phyla.

Virions produced by prophages can be captured during viral-particle enrichment steps as part of viral metagenome preparation. Thus, predictions of the PhiSpy algorithm were tested by determining whether viral metagenome reads could be recruited to sequences of all 170 of the predicted prophage regions. Because
104 of the 170 DGR-containing lysogens belonged to the Bacteroidetes or Firmicutes phyla (Additional file 2), whose members dominate the human gastrointestinal tract [17], publicly available viral metagenomes from human skin and fecal samples were downloaded $(n=1,366)$. Approximately three billion quality-filtered sequence reads were concatenated into a single file and aligned to each predicted prophage region (Fig. 1). Ninety-two of the 170 regions recruited viral reads at $>10 \times$ mean fold coverage, demonstrating the presence of an integrated phage capable of producing virions (Additional file 2). Predicted prophage regions contained identifiable phage genes, such as capsids, tails, and integrases, validating the recruitment of reads to a temperate phage (Additional file 3). Through the bioinformatic approach presented here, 92 DGRs were classified as viral in origin.

All of the phages possessing a DGR, including BPP-1 and the two Vibrio phages, exhibit a temperate lifestyle $[4,18,19]$. To determine if this observation was an artifact of temperate phages being overrepresented in the RefSeq, PhagesDB, and prophage collection, all phage life histories were classified using PHACTS [20]. The majority of phages in these databases could not have a lifestyle assigned $(n=2,911)$ (Additional file 4 ). Where a lifestyle could be confidently assigned, 1,181 phages were predicted to be temperate and 332 were predicted to be lytic. However, despite the database bias towards temperate phages, the observed number of DGRs in temperate phages was still higher than expected $\left(\chi^{2}, * P=0.0012\right)$. Therefore, DGRs are preferentially encoded by temperate phages.

\section{Bacteroides dorei is lysogenized by a DGR-containing temperate phage}

Several regions predicted to contain a DGR-encoding prophage recruited reads from multiple independent studies (Additional file 5), which was unexpected given the high interpersonal diversity of human viromes [21]. A 66-kb region in Bacteroides dorei was further examined because it recruited reads from 13 different human viral studies with a mean fold coverage of $229 \times$ (Fig. 2a). The reads aligned to a discrete $43 \mathrm{~kb}$ locus encoding 45 ORFs. Seven ORFs with significant homology to known phage structural proteins could be annotated, including two tail proteins, a baseplate, portal, neck, and two capsid genes (Fig. 2b and Additional file 6). Genes required for phage genome packaging and integration were detected, including a large and small terminase and phage Mu-like transposase. The DGR cassette was composed of an RT with two adjacent $117 \mathrm{bp}$ repeats that were mismatched at 14 positions corresponding to adenine bases. Proximal to the RT gene was a small ORF whose amino acid sequence had a similar isoelectric point and molecular weight as the accessory variability determinant of 


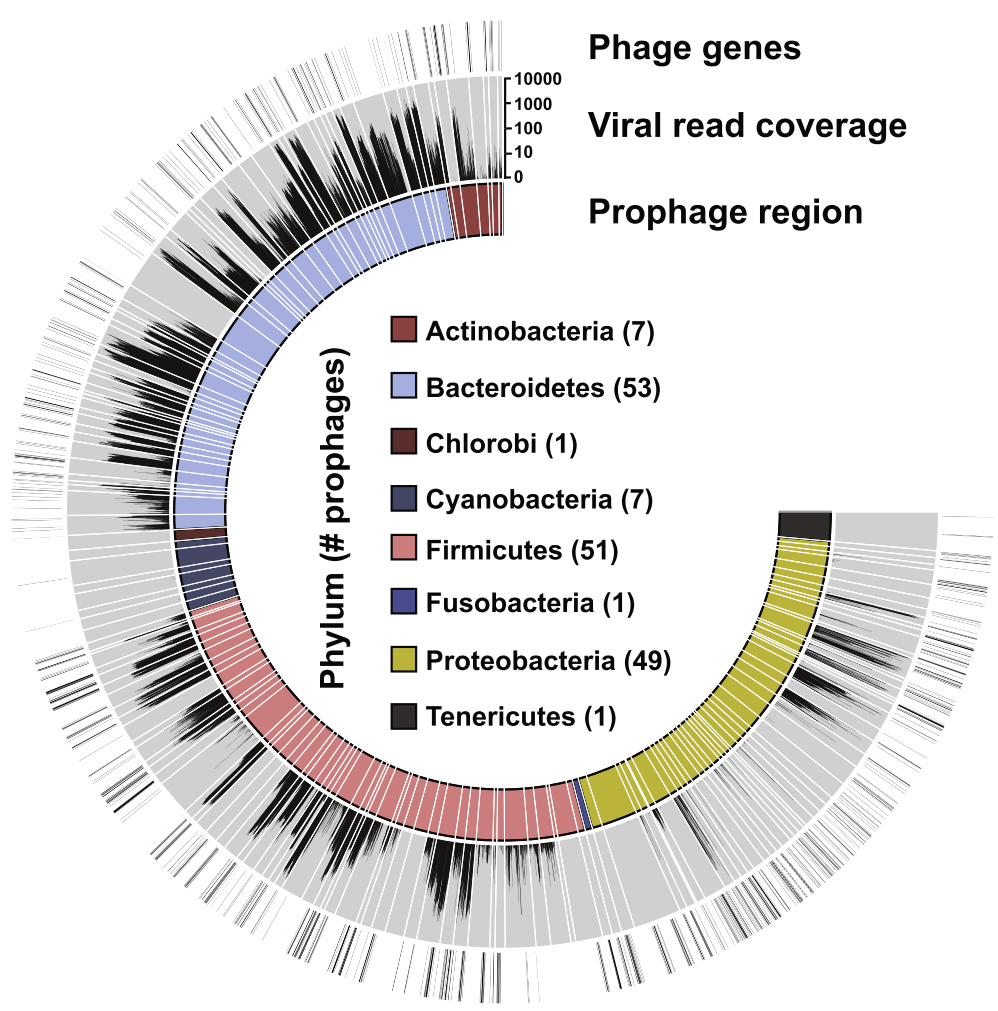

Fig. 1 DGRs in bacteria are encoded by functional prophages. Predicted prophage-containing regions with a DGR $(n=170)$ are depicted on the inner ring and colored according to host phylum. Each predicted prophage-containing region was used as a reference to align sequencing reads from human viromes. The coverage plot of reads for each region is shown on the middle ring. ORFs whose annotations contained the keyword "phage" are indicated with a hash mark on the outer ring. Sequences of all predicted prophage-containing regions and their annotations are available in Additional files 11 and 3, respectively

BPP-1 [4]. The VR was located at the $\mathrm{C}$ terminus (residues 2188-2225) of a large ORF possessing a C-type lectin domain. Collectively, these features indicated the $43 \mathrm{~kb}$ region was a prophage genome that harbored a DGR cassette.

Phage induction assays on a culture of $B$. dorei were performed to validate mature virion production in vitro. A culture of $B$. dorei was obtained and cultured anaerobically at $37{ }^{\circ} \mathrm{C}$. The culture was treated with the antibiotic carbadox, a known prophage inducing agent [22-24]. After $10-12 \mathrm{~h}$ of anaerobic growth, bacterial cells were removed. Transmission electron microscopy identified a virus with a $200 \mathrm{~nm}$ flexible tail typical of the Siphoviridae (Fig. 2c). Lastly, we confirmed the observed phage contained the predicted prophage genotype using PCR (Fig. 2d). This phage was named Bacteroides dorei Hankyphage and exemplifies the power of the bioinformatic pipeline to identify inducible prophages, including those that encode DGRs.

\section{Hankyphage exhibits broad-host-range and global distribution}

Plaque assays combining a cell-free phage lysate and susceptible bacterial hosts can determine the minimal host range of a phage [25]. A phage lysate was prepared from an induced culture of $B$. dorei and combined with a naïve strain of Bacteroides dorei in top agar under anaerobic conditions. Lawns of the naïve strain or additional Bacteroides spp. did not yield observable plaques (data not shown). As an alternative, the NCBI RefSeq database of bacterial genomes (accessed 07/2017) was queried for the Hankyphage genome. The complete, $43 \mathrm{~kb}$ Hankyphage genome was present in 13 unique Bacteroides species at $\geq 95 \%$ nucleotide identity (Fig. 3a). Consistent with $\mathrm{Mu}$-like transposition, the site of integration was random and flanked by 5 bp direct repeats [26] (Fig. 3a). Thus, Hankyphage exhibits broad-host-range and each tropic variant was assigned a unique indication (Hankyphage p00-Hankyphage p12).

To test if the DGR cassette diversifies the variable repeat in different Bacteroides hosts, the TRs and cognate VRs of each tropic variant were extracted for multiple alignments (Clustal Omega). The aligned TRs contained no substitutions while the VRs exhibited adenine-specific substitutions in asparagine codons, a distinguishing attribute of DGR-mediated variation [4] (Fig. 3b). None of the tropic variants shared the same VR sequence. Interestingly, two tropic variants (B. dorei and B. cellulosilyticus) 


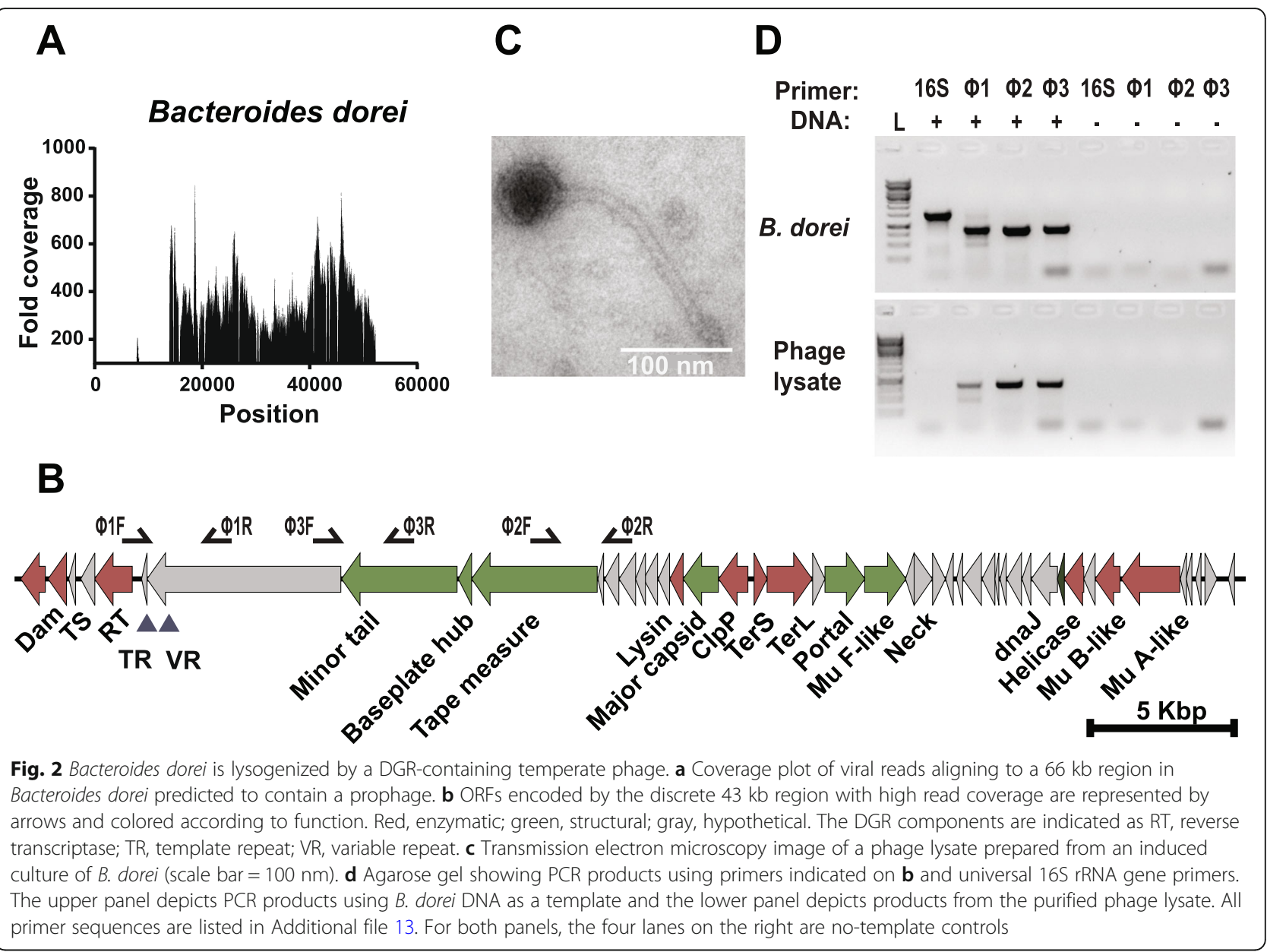

only differed in the sequence of their VR along the entirety of their aligned genomes. The VR contained 21 variable positions, enabling Hankyphage to explore a sequence space of potentially $10^{12}$ unique variants. Despite the similar capacity for sequence variation as the BPP-1 tail fiber, Hankyphage's DGR target protein shared no sequence similarity with any characterized protein (Additional file 6).

To estimate the ubiquity of Hankyphage in the environment, total-community metagenomes and viromes available on the NCBI SRA were searched for reads matching the Hankyphage genome. An untargeted selection of 37,583 total-community metagenomes from the NCBI SRA (accessed 03/2018) spanning diverse microbial environments was curated previously [27]. In total, 12,774 metagenomes contained $>1$ read aligning to the Hankyphage genome $(12,774 / 37,583,34 \%)$ (Fig. 4 and Additional file 7). The same analysis was repeated using the previous database of 1,366 human-associated viromes sampled from geographically separated individuals. Sequencing reads recruited at $>97 \%$ nucleotide identity could be recovered from 221 of 1,366 viromes (Additional file 8). All 221 viromes were derived from human fecal samples (221/1038) while none of the human skin virome reads were recruited (0/329). For a given virome sample, Hankyphage was present at $4 \times$ $10^{-8}$ to $4 \times 10^{-2}$ fractional abundance (Additional file 8). Using these data to estimate the number of Hankyphage virions, approximately $3.3 \times 10^{17}$ exist globally (Additional file 9). In summary, Hankyphage is present in microbial environments, namely the human gastrointestinal tract, from locations sampled around the world.

\section{Function of hypervariable proteins}

To assess the functional role of all the DGR cassettes identified, structures of the variable repeat-containing ORFs were predicted using Phyre2 [28]. Five of the ORFs exhibited structural homology to the major tropism determinant of phage BPP-1, signifying these phages may use a similar strategy as BPP-1 to mutagenize their tail fibers and broaden host range (Additional file 10) [4]. None of the other hypervariable proteins were homologous to known phage proteins; however, 57 hypervariable genes were homologous to a DGR target protein from Thermus aquaticus and 29 were homologous to a 


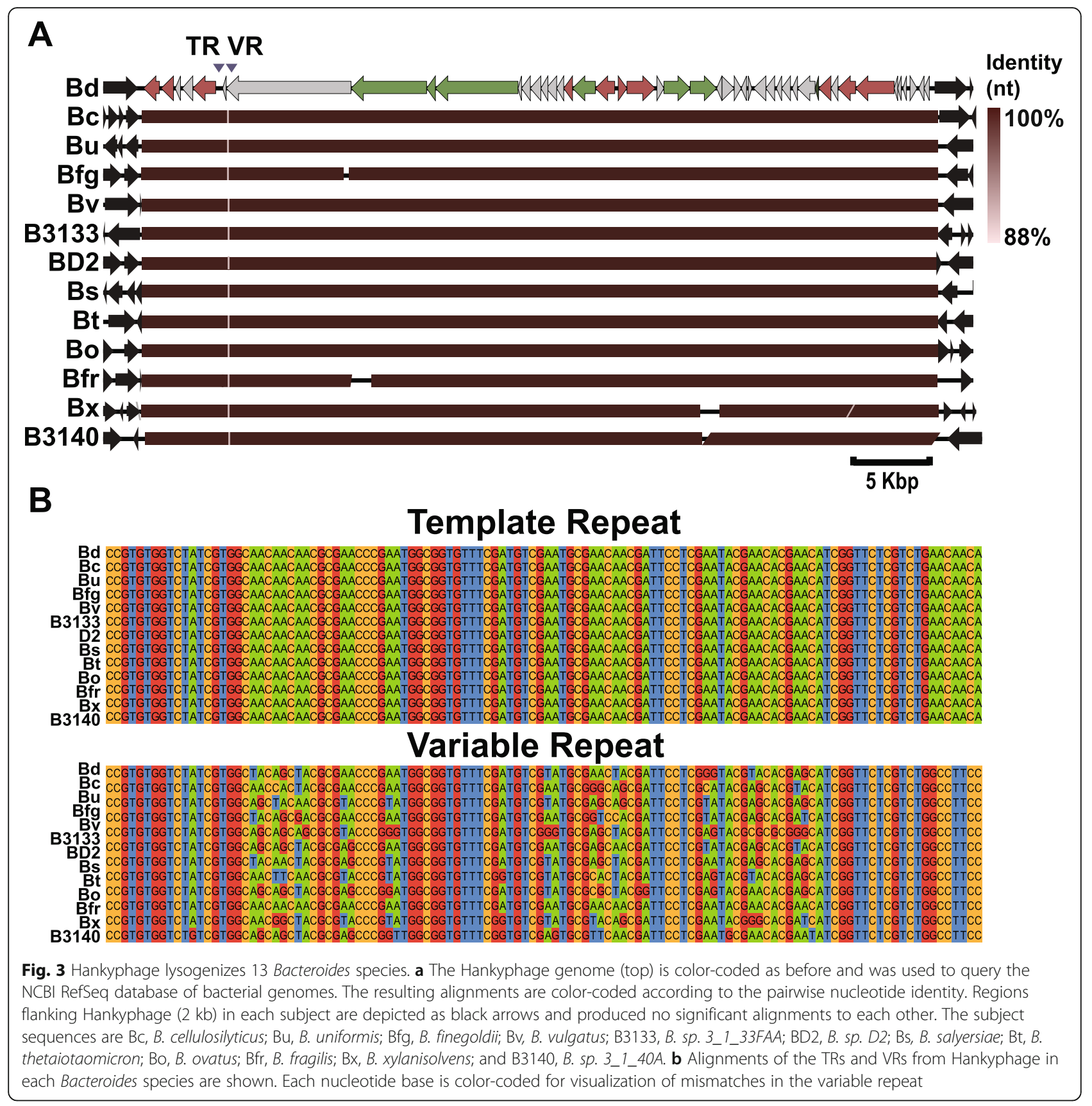

pilin tip from Bacteroides ovatus. The pilin structure was recently shown to contain a C-type lectin domain, supporting previous observations that this fold is a conserved target of DGRs [13, 29]. Overall, the functional roles of these hypervariable proteins remain to be determined.

\section{Discussion}

By combining a prophage prediction algorithm with mapping reads from viromes, 92 phage-encoded DGRs were identified. Many of the proteins targeted for diversification contain a subtype of the C-type lectin fold, suggesting a role in binding interactions with diverse ligands. Phage DGR proteins lack detectible transmembrane regions or signal peptides found on surfacedisplayed bacterial DGR proteins $[13,30]$ (Additional file 10). While this supports their phage origin, discerning whether the DGR target proteins are components of functional prophages or defective remnants is difficult. Here, a generalized bioinformatic pipeline was employed to identify functional prophages where viral read data were available.

In one case, an integrated prophage from $B$. dorei was induced, thereby verifying the bioinformatic prediction. This temperate bacteriophage, Hankyphage, is the fifth 


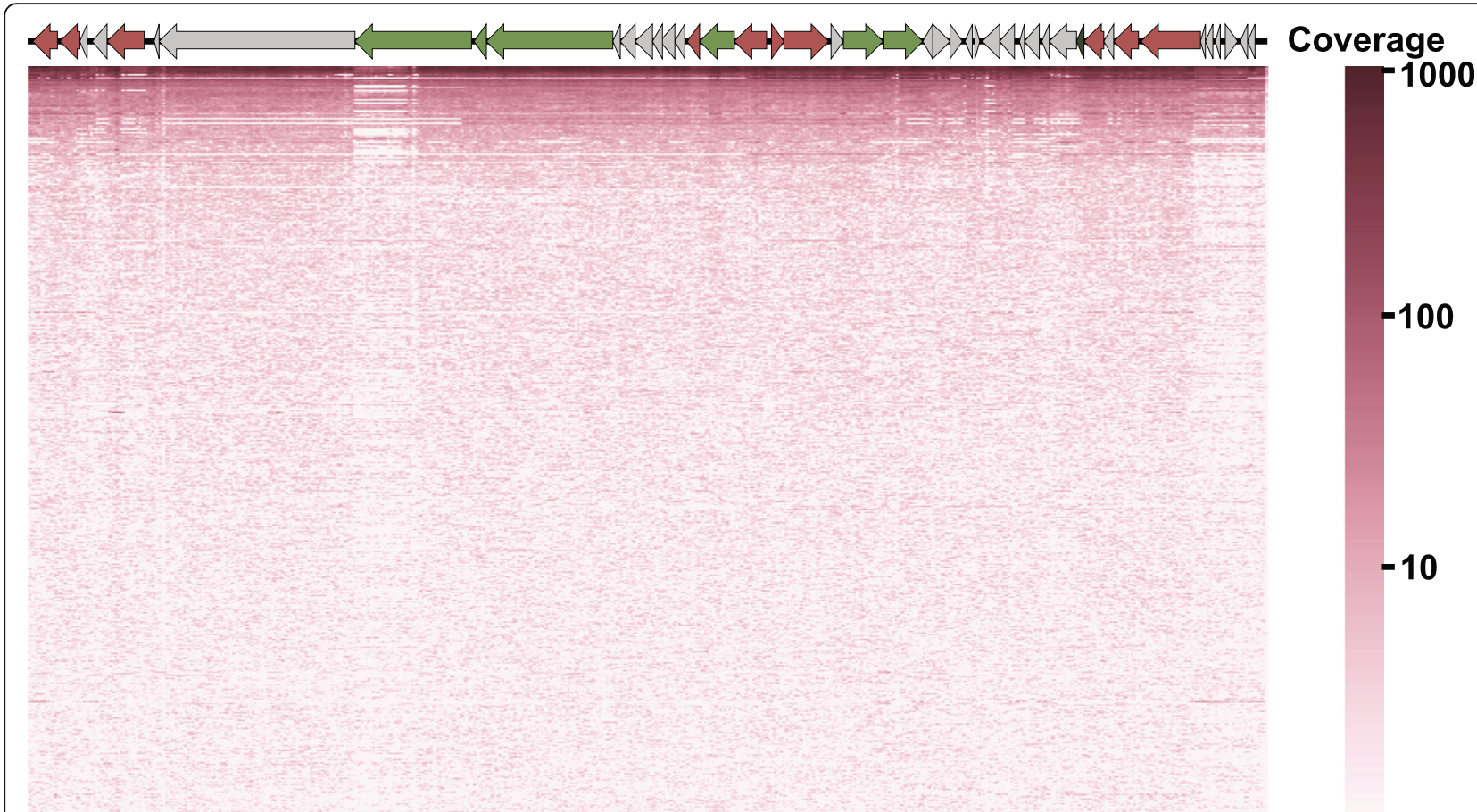

Fig. 4 Coverage plot of the Hankyphage genome in metagenomes. Each row is a metagenome $(n=500)$. The $x$-axis of the heat map displays the 42,831 bp length of the Hankyphage genome. The color bar is scaled proportionally to the coverage of each nucleotide in the Hankyphage genome. Details of the read alignments for all metagenomes are available in Additional file 7

phage to be induced that possesses a DGR. Hankyphage was detected as a complete prophage in 13 different Bacteroides species at $>95 \%$ nucleotide identity. Furthermore, Hankyphage's complete genome was recovered in whole-community and viral metagenomes. These observations suggest that Hankyphage can infect a wide-range of Bacteroides in diverse environments despite not infecting naïve Bacteroides hosts in vitro. Additional factors may be required for a productive infection outside the gastrointestinal tract. Previous analyses of intestinal viral communities demonstrate few genotypes are shared between individuals [21]. However, analysis of metagenomes generated from human fecal samples collected around the globe discloses that Hankyphage resides in the gastrointestinal tract of approximately $50 \%$ of the human population (Additional file 9).

Despite there being hundreds of lytic phage genomes in public databases, DGRs were only identified in temperate phages. Given that the global phage diversity far exceeds that of cultured isolates, identification of a DGR-containing lytic phage may be forthcoming [31]. Nevertheless, DGR-diversification may provide increased functionality for temperate phages. Targeted mutagenesis of phage genes would offset accurate replication by bacterial DNA polymerases, poising temperate phages for dynamic responses to changing environmental conditions. That the majority of DGR-containing phages lysogenize human gut commensals suggests that DGRs are advantageous in host-associated environments. Similarly, DGRs are enriched in host-associated bacteria and archaea with reduced genome sizes, highlighting the parallel emergence of hypervariability cassettes in host-associated environments [10].

The variable repeats of known DGRs lie within C-type lectin folds and Ig-like domains [2, 12]. Both C-type lectins and Ig-like domains are encoded by phages, including Hankyphage and two other Bacteroides phages [32-34]. In phage $\mathrm{T} 4$, the Ig-like domain-containing protein $\mathrm{Hoc}$ mediates phage adherence to mucus, thereby increasing the rate of T4 adsorption onto E. coli host cells [35]. Consistent with this bacteriophage adherence to mucus model, Hankyphage may employ a similar strategy. By utilizing a C-type lectin to adhere to mucus, Hankyphage may increase the rate of encounter with Bacteroides hosts in mucus. Targeted hypervariation in the C-type lectin could modulate phage diffusion in mucus, enabling optimal search strategies in different mucosal environments.

\section{Conclusions}

In this study, we present evidence that DGR cassettes are encoded by temperate bacteriophages integrated in the chromosomes of bacteria as prophages. Many of these DGRs were previously considered to be bacterial in origin. While the functions of these hypervariable 
phage proteins remain enigmatic, this research will enable future dissection of their roles in phage life cycles. Moreover, the isolation of a single phage shared amongst an estimated one-half of the human population is an exception to our current understanding that the human viral community is largely composed of unique viruses.

\section{Methods}

\section{Identification of DGRs}

Three databases were searched for DGR cassettes. The first database contained bacteriophage genomes available on the RefSeq database dereplicated at $98 \%$ identity using CD-HIT ( $n=1881$, accessed 06/2016) [36, 37]. A second database of actinobacteriophage genomes ( $n=2322$, accessed $12 / 2017)$ was downloaded from the PhagesDB website [38]. A third database of 31,946 predicted prophages was generated from 11,278 bacterial and archaeal genomes using the program PhiSpy [15]. All predicted prophage sequences are available from [39]. Sequences in each database were screened for reverse transcriptases (RTs) (Pfam model PF00078) using HMMer [40]. Sequences with RTs were subsequently scanned for template repeats (TRs) and variable repeats (VRs) using the program DiGRef with default settings (minimum 10 adenines in TR, minimum 7 adenine substitutions in VR) modified to accept local inputs [8]. Template repeat and variable repeat alignments were made using Clustal Omega and visualized with JalView [41, 42]. Life history assignments for phages in the databases (RefSeq, PhagesDB, and the DGR-containing predicted prophage regions) were accomplished using PHACTS [20]. A lifestyle assignment was considered confident if the average score of the trees minus the standard deviation was $>0.5$. Otherwise, no life history assignment was made.

\section{Viral read mapping to predicted prophage-containing regions}

A collection of human-associated viromes available from the NCBI sequence read archive (SRA) was previously curated [31]. Additional human viromes were added to the database and all accessions are provided (Additional file 8). All of these viromes were downloaded from the SRA using the fastq-dump utility, quality filtered and dereplicated with Prinseq employing the following flags: derep 12345 -lc_method entropy -lc_threshold 50 -trim_qual_left 15 -trim_qual_right 15 -trim_qual_type mean -trim_qual_rule $1 \mathrm{t}$-trim_qual_window 2 -trim_tail_left 5 -trim_tail_right 5 -min_len 60 -min_qual_mean 15 -ns_max_p 1 [43]. Reads from each virome were concatenated into a single file and mapped to predicted prophage-containing regions harboring a DGR $(n=170$, Additional file 11) using Bowtie2 with default parameters [44]. Coverage was calculated with Samtools and visualized using Anvio [45, 46]. To calculate the fractional abundance of Bacteroides dorei Hankyphage in each sample, reads from each virome were mapped to the Hankyphage genome at 97\% identity using SMALT [47]. The fractional abundance of Hankyphage in each virome was calculated as previously described [31].

\section{Prophage genome annotation}

Open reading frames (ORFs) in predicted prophage-containing regions were called by Prodigal and annotated by BLASTp searches against the NCBI COG database, where hits are considered significant if the $e$ value is $<10^{-5}[48,49]$. Additional annotations were determined by HMM searches against the Pfam-A database with a bitscore cutoff of $>25$ [50, 51]. ORFs containing a variable repeat were analyzed using Phyre 2 and Phobius [28, 52]. To identify the host range of Hankyphage, the phage genome (Additional file 12) was used as a query against all subject Bacteroides genomes available on RefSeq using BLASTn (accessed 06/2017). Query-subject alignments were visualized using Easyfig [53].

\section{Metagenome read mapping to Hankyphage}

A collection of whole-community metagenomes available from the NCBI SRA was previously curated [27]. An untargeted selection of 37,583 metagenomes was downloaded using the fastq-dump utility of the SRA toolkit and mapped to the Hankyphage genome using Bowtie2 with default parameters [54-56]. Binary Alignment Map (BAM) files were sorted and indexed using Samtools version 1.7 [46]. Heatmaps were generated from the BAM files using publicly available scripts [57].

\section{Phage induction, PCR, and transmission electron microscopy}

A culture of Bacteroides dorei strain CL02T12C06 (HM-719) was obtained through BEI Resources, NIAID, $\mathrm{NIH}$ as part of the Human Microbiome Project. B. dorei was maintained on supplemented brain heart infusion media (BHIS) [38 g/L BHI, $5 \mathrm{~g} / \mathrm{L}$ yeast extract, $1.5 \mathrm{~g} / \mathrm{L}$ L-cysteine, $1 \mathrm{mM} \mathrm{CaCl} 2,10 \mathrm{mM} \mathrm{MgSO}_{4}$, $1 \mu \mathrm{g} / \mathrm{L}$ vitamin $\mathrm{K} 3,0.24 \mu \mathrm{g} / \mathrm{L}$ histidine-hematin]. BHIS broth was inoculated with a glycerol stock of $B$. dorei and cultured anaerobically at $37{ }^{\circ} \mathrm{C}$. Early-log cultures were induced for prophage with $8 \mu \mathrm{g} / \mathrm{mL}$ carbadox and harvested after $8-10 \mathrm{~h}$ of growth. The cultures were clarified of bacterial cells through chloroform treatment and syringe-driven $0.22 \mu \mathrm{m}$ filtration. The clarified supernatant was stored at $4{ }^{\circ} \mathrm{C}$ over 0.1 volumes chloroform and used for downstream assays. For PCR, the phage lysate was treated with $3 \mathrm{U}$ of DNase I and incubated for $1 \mathrm{~h}$ at $37{ }^{\circ} \mathrm{C}$, 
followed by heat inactivation for $10 \mathrm{~min}$ at $99{ }^{\circ} \mathrm{C}$. Primer sequences are listed in Additional file 13. For transmission electron microscopy, $30 \mu \mathrm{l}$ of the purified phage lysate was added to glow-discharged 300-mesh copper grids coated with $10 \mathrm{~nm}$ formvar and $1 \mathrm{~nm}$ carbon (Electron Microscopy Sciences, PA) for $3 \mathrm{~min}$. To remove the salts in the buffer, the grids were rinsed three times by drops of distilled water $(20 \mu \mathrm{l})$. The grids were then negatively stained with uranyl acetate $(0.5 \%)$ for $15 \mathrm{~s}$, dried and examined with a FEI Tecnai T12 TEM (FEI, Hillsboro, OR) operating at $120 \mathrm{kV}$ at the SDSU Electron Microscopy Facility. Micrographs were taken by an AMT HX41 side-mounted digital camera (Advanced Microscopy Technique, Woburn, MA).

\section{Additional files}

Additional file 1: DGRs identified in isolated phages from the RefSeq database. For each DGR, annotation of the VR-encoding ORF was made using Phyre2. A domain analysis was conducted using HMMer against the Pfam-A database. Lifestyle assignment was extracted from the literature. (XLSX $9 \mathrm{~kb}$ )

Additional file 2: Nucleotide coordinates, taxonomy, and coverage of the DGR cassettes. The nucleotide coordinates of the reverse transcriptase, template repeat and variable repeat on the predicted prophage-containing region are listed in columns A-H (only the first TR/ VR pair is reported). Where possible, the nucleotide coordinates of the prophage region in the RefSeq database is listed in columns $\mathrm{H}-$ J, along with the taxonomic details of the host organism in columns $\mathrm{K}-\mathrm{R}$. The mean coverage of mapped sequencing reads against each predicted prophage is reported in column U. (XLSX $41 \mathrm{~kb}$ )

Additional file 3: ORFs in predicted prophage-containing regions. ORFs in all predicted prophage-containing regions were called by Prodigal and assigned a unique id. The nucleotide coordinates and sequence of each ORF is provided. For significant hits, the description and accession of each COG category and Pfam-A family is listed. (XLSX $3960 \mathrm{~kb}$ )

Additional file 4: Proportion of temperate and lytic viruses in the phage databases (RefSeq, PhagesDB, and DGR-containing prophage collection). Each phage life history was classified using PHACTS as 'Iytic' or 'temperate' if the trees voting for that lifestyle is $>0.5$. Otherwise, the phage lifestyle is listed as 'N/A'. (XLSX $118 \mathrm{~kb}$ )

Additional file 5: Predicted prophage-containing regions with a DGR ( $n$ $=30$ ) that recruit reads from multiple human virome studies. Each predicted prophage-containing region was used as a reference to align sequencing reads from human viromes. The coverage plot of recruited reads from each study are shown on the middle rings, listed according to the first author of the study (log scale, 0 - 1000x fold coverage). (PDF $2472 \mathrm{~kb}$ )

Additional file 6: ORFs encoded in the Hankyphage genome. Functional annotations for each ORF were determined by comparisons to the Pfam-A, Phyre2, or conserved domain database. For significant hits, the database and accession to the hit are listed with the corresponding $e$-value or confidence score. (XLSX $12 \mathrm{~kb}$ )

Additional file 7: Metagenomic survey of the SRA for Hankyphage. The Hankyphage genome was used as a reference to align reads from wholecommunity metagenomes using Bowtie2. The number of aligned reads and percent coverage of the Hankyphage genome is reported. Where possible, the abstract from each study is provided. (XLSX $947 \mathrm{~kb}$ )

Additional file 8: Fractional abundance of Hankyphage in viromes. The Hankyphage genome was used as a reference to align reads from each human virome at $97 \%$ identity. For each virome containing $>1$ aligned read $(n=221)$, the fractional abundance of Hankyphage was calculated
[31]. The related metadata for each virome was extracted from the SRA database and listed. (XLSX $78 \mathrm{~kb}$ )

Additional file 9: Calculation of the global number of Hankyphage virions. (DOCX $37 \mathrm{~kb}$ )

Additional file 10: Annotation of the VR-containing ORFs in DGRs. All ORFs with a VR were annotated using Phyre 2 and HMM searches against the Pfam-A database. Annotations were only considered significant if the Phyre2 confidence is $>97$ or HMMer bitscore is $>25$. Otherwise, the annotation is listed as N/A. The presence or absence of transmembranespanning regions and signal peptides were identified using Phobius and indicated as "yes," "no," and "signal peptide," respectively. (XLSX 17 kb)

Additional file 11: Sequences of all 170 predicted prophage-containing regions. (FASTA $11271 \mathrm{~kb}$ )

Additional file 12: Sequence of Hankyphage. (GBK $81 \mathrm{~kb}$ ) Additional file 13: Primers used in the study (written $5^{\prime}$ to $3^{\prime}$ ). (DOCX $22 \mathrm{~kb}$ )

\section{Acknowledgements}

The authors acknowledge the SDSU Electron Microscopy Facility for assistance with sample analysis. We are grateful to Giselle Cavalcanti and Heather Maughan for careful reading of the manuscript. S.B. acknowledges funding and support from San Diego State University.

\section{Funding}

This work was supported by NSF Partnerships for International Research and Education (PIRE) Grant (124351; F.R.). Searching the SRA was supported by XSEDE allocation TG-MCB170036 to Robert Edwards.

\section{Availability of data and materials}

The Genbank-formatted sequence of Hankyphage is provided in Additional file 13 . Sequences and annotations of all 170 DGR-containing predicted prophage regions are provided as additional files. The metagenomes and viromes analyzed in the study are publicly available on the NCBI SRA database and all accessions are listed in Additional files 7 and 8. BAM files are available upon request.

\section{Authors' contributions}

SB performed the investigation and formal analysis and drafted the manuscript. ACG performed the data curation. KM designed and implemented the software. $\mathrm{KL}$ visualized the data. SH participated in the investigation. RE designed the software and coordinated the project. FR conceived and supervised the project. All authors read and approved the final manuscript.

Ethics approval and consent to participate

Not applicable

\section{Consent for publication}

Not applicable

\section{Competing interests}

The authors declare that they have no competing interests.

\section{Publisher's Note}

Springer Nature remains neutral with regard to jurisdictional claims in published maps and institutional affiliations.

\section{Author details}

'Department of Biology, San Diego State University, 5500 Campanile Drive, San Diego, CA 92182, USA. '2Department of Computer Science, San Diego

State University, 5500 Campanile Drive, San Diego, CA 92182, USA.

Received: 9 August 2018 Accepted: 8 October 2018

Published online: 23 October 2018

\section{References}

1. Minot S, Bryson A, Chehoud C, Wu GD, Lewis JD, Bushman FD. Rapid evolution of the human gut virome. Proc Natl Acad Sci. 2013;110:12450-5. https://doi.org/10.1073/pnas.1300833110. 
2. Minot S, Grunberg S, Wu GD, Lewis JD, Bushman FD. Hypervariable loci in the human gut virome. Proc Natl Acad Sci. 2012;109:3962-6. https://doi.org/ 10.1073/pnas.1119061109.

3. Hannigan GD, Zheng Q, Meisel JS, Minot SS, Bushman FD, Grice EA. Evolutionary and functional implications of hypervariable loci within the skin virome. PeerJ. 2017;5:e2959. https://doi.org/10.7717/peerj.2959.

4. Liu M, Deora R, Doulatov SR, Gingery M, Eiserling FA, Preston A, et al. Reverse transcriptase - mediated tropism switching in Bordetella. Science. 2002;295:2091-5

5. Naorem SS, Han J, Wang S, Lee WR, Heng X, Miller JF, et al. DGR mutagenic transposition occurs via hypermutagenic reverse transcription primed by nicked template RNA. Proc Natl Acad Sci. 2017;114:E10187-95. https://doi. org/10.1073/pnas.1715952114.

6. Doulatov S, Hodes A, Dai L, Mandhana N, Liu M, Deora R, et al. Tropism switching in Bordetella bacteriophage defines a family of diversitygenerating retroelements. Nature. 2004;431:476-81.

7. Miller JL, Le Coq J, Hodes A, Barbalat R, Miller JF, Ghosh P. Selective ligand recognition by a diversity-generating retroelement variable protein. PLoS Biol. 2008;6:1195-207.

8. Schillinger T, Lisfi M, Chi J, Cullum J, Zingler N. Analysis of a comprehensive dataset of diversity generating retroelements generated by the program DiGReF. BMC Genomics. 2012;13:430. https://doi.org/10.1186/1471-2164-13-430.

9. Paul BG, Bagby SC, Czornyj E, Arambula D, Handa S, Sczyrba A, et al. Targeted diversity generation by intraterrestrial archaea and archaeal viruses. Nat Commun. 2015;6:6585. https://doi.org/10.1038/ncomms7585.

10. Paul BG, Burstein D, Castelle CJ, Handa S, Arambula D, Czornyj E, et al. Retroelement-guided protein diversification abounds in vast lineages of bacteria and archaea. Nat Microbiol. 2017;2:17045. https://doi.org/10.1038/ nmicrobiol.2017.45.

11. Simon DM, Zimmerly S. A diversity of uncharacterized reverse transcriptases in bacteria. Nucleic Acids Res. 2008:36:7219-29.

12. Wu L, Gingery M, Abebe M, Arambula D, Czornyj E, Handa S, et al. Diversitygenerating retroelements: natural variation, classification and evolution inferred from a large-scale genomic survey. Nucleic Acids Res. 2017; December:1-14. https://doi.org/10.1093/nar/gkx1150.

13. Le Coq J, Ghosh P. Conservation of the C-type lectin fold for massive sequence variation in a Treponema diversity-generating retroelement. Proc Natl Acad Sci. 2011:108:14649-53.

14. Kang HS, McNair K, Cuevas D, Bailey B, Segall A, Edwards RA. Prophage genomics reveals patterns in phage genome organization and replication. bioRxiv. 2017:1-28. https://doi.org/10.1101/114819.

15. Akhter S, Aziz RK, Edwards RA. PhiSpy: a novel algorithm for finding prophages in bacterial genomes that combines similarity- and compositionbased strategies. Nucleic Acids Res. 2012:40:1-13.

16. Cornuault JK, Petit M-A, Mariadassou M, Benevides L, Moncaut $E$, Langella $P$, et al. Phages infecting Faecalibacterium prausnitzii belong to novel viral genera that help to decipher intestinal viromes. Microbiome. 2018;6:65. https://doi.org/10.1186/s40168-018-0452-1.

17. Huttenhower C, Fah Sathirapongsasuti J, Segata N, Gevers D, Earl AM, Fitzgerald $M G$, et al. Structure, function and diversity of the healthy human microbiome. Nature. 2012;486:207-14. https://doi.org/10.1038/nature11234

18. Oakey HJ, Cullen BR, Owens L. The complete nucleotide sequence of the Vibrio harveyi bacteriophage VHML. J Appl Microbiol. 2002;93:1089-98. https://doi.org/10.1046/j.1365-2672.2002.01776.x.

19. Zabala B, Hammerl JA, Espejo RT, Hertwig S. The linear plasmid prophage Vp58.5 of Vibrio parahaemolyticus is closely related to the integrating phage VHML and constitutes a new incompatibility group of telomere phages. J Virol. 2009;83:9313-20. https://doi.org/10.1128/JVI.00672-09.

20. McNair K, Bailey BA, Edwards RA. PHACTS, a computational approach to classifying the lifestyle of phages. Bioinformatics. 2012;28:614-8.

21. Reyes A, Haynes M, Hanson N, Angly FE, Heath AC, Rohwer F, et al. Viruses in the faecal microbiota of monozygotic twins and their mothers. Nature. 2010;466:334-8. https://doi.org/10.1038/nature09199.

22. Kohler B, Karch H, Schmidt H. Antibacterials that are used as growth promoters in animal husbandry can affect the release of Shiga-toxin-2 converting bacteriophages and Shiga toxin 2 from Escherichia coli strains. Microbiology. 2000;146:1085-90.

23. Johnson TA, Looft T, Severin AJ, Bayles DO, Nasko DJ, Wommack KE, et al. The in-feed antibiotic Carbadox induces phage gene transcription in the swine gut microbiome. MBio. 2017:8:e00709-17. https://doi.org/10.1128/ mBio.00709-17.
24. Bearson BL, Allen HK, Brunelle BW, Lee IS, Casjens SR, Stanton TB. The agricultural antibiotic carbadox induces phage-mediated gene transfer in Salmonella. Front Microbiol. 2014;5:1-8.

25. Adams MH. Bacteriophages. New York: Interscience Publishers, Inc.; 1959.

26. Allet B. Mu insertion duplicates a 5 base pair sequence at the host inserted site. Cell. 1979;16:123-9.

27. Torres PJ, Edwards RA, McNair KA. PARTIE: a partition engine to separate metagenomic and amplicon projects in the sequence read archive. Bioinformatics. 2017;33:2389-91.

28. Kelly LA, Mezulis S, Yates C, Wass M, Sternberg M. The Phyre2 web portal for protein modelling, prediction, and analysis. Nat Protoc. 2015;10:845-58. https://doi.org/10.1038/nprot.2015-053.

29. Xu Q, Shoji M, Shibata S, Naito M, Sato K, Elsliger MA, et al. A distinct type of pilus from the human microbiome. Cell. 2016;165:690-703.

30. Arambula D, Wong W, Medhekar BA, Guo H, Gingery M, Czornyj E, et al. Surface display of a massively variable lipoprotein by a legionella diversitygenerating retroelement. Proc Natl Acad Sci U S A. 2013;110:8212-7. https:// doi.org/10.1073/pnas.1301366110.

31. Cobián Güemes AG, Youle M, Cantú VA, Felts B, Nulton J, Rohwer F. Viruses as winners in the game of life. Annu Rev Virol. 2016;3:197-214. https://doi. org/10.1146/annurev-virology-100114-054952.

32. Reyes A, Wu M, McNulty NP, Rohwer FL, Gordon Jl. Gnotobiotic mouse model of phage-bacterial host dynamics in the human gut. Proc Natl Acad Sci. 2013;110:20236-41. https://doi.org/10.1073/pnas.1319470110.

33. Dutilh BE, Cassman N, McNair K, Sanchez SE, Silva GGZ, Boling L, et al. A highly abundant bacteriophage discovered in the unknown sequences of human faecal metagenomes. Nat Commun. 2014;5:1-11. https://doi.org/10. 1038/ncomms5498.

34. Fraser JS, Yu Z, Maxwell KL, Davidson AR. Ig-like domains on bacteriophages: a tale of promiscuity and deceit. J Mol Biol. 2006;359: 496-507. https://doi.org/10.1016/j.jmb.2006.03.043.

35. Barr JJ, Auro R, Sam-Soon N, Kassegne S, Peters G, Bonilla N, et al. Subdiffusive motion of bacteriophage in mucosal surfaces increases the frequency of bacterial encounters. Proc Natl Acad Sci. 2015;112:13675-80. https://doi.org/10.1073/pnas.1508355112.

36. Fu L, Niu B, Zhu Z, Wu S, Li W. CD-HIT: accelerated for clustering the nextgeneration sequencing data. Bioinformatics. 2012;28:3150-2.

37. Brister JR, Ako-Adjei D, Bao Y, Blinkova O. NCBI viral Genomes resource. Nucleic Acids Res. 2015;43:D571-7.

38. Russell DA, Hatfull GF. PhagesDB: the actinobacteriophage database. Bioinformatics. 2017;33:784-6.

39. Kang HS. Phispy prophage predictions. Figshare 2017. https://figshare.com/ articles/PhiSpy_prophage_predictions/3146656. Accessed 1 June 2017.

40. Finn RD, Clements J, Eddy SR. HMMER web server: interactive sequence similarity searching. Nucleic Acids Res. 2011;39:29-37.

41. Waterhouse AM, Procter JB, Martin DMA, Clamp M, Barton GJ. Jalview version 2-a multiple sequence alignment editor and analysis workbench. Bioinformatics. 2009;25:1189-91.

42. Sievers F, Wilm A, Dineen D, Gibson TJ, Karplus K, Li W, et al. Fast, scalable generation of high-quality protein multiple sequence alignments using Clustal omega. Mol Syst Biol. 2011;7:539.

43. Schmieder R, Edwards R. Quality control and preprocessing of metagenomic datasets. Bioinformatics. 2011;27:863-4.

44. Langmead B, Salzberg SL. Fast gapped-read alignment with bowtie 2. Nat Methods. 2012:9:357-9.

45. Eren AM, Esen ÖC, Quince C, Vineis JH, Morrison HG, Sogin ML, et al. Anvi'o: an advanced analysis and visualization platform for 'omics data. PeerJ. 2015; 3:e1319. https://doi.org/10.7717/peerj.1319.

46. Li H, Handsaker B, Wysoker A, Fennell T, Ruan J, Homer N, et al. The sequence alignment/map format and SAMtools. Bioinformatics. 2009;25: 2078-9.

47. Ponstingl H. SMALT. 2010. http://www.sanger.ac.uk/science/tools/smalt-0.

48. Galperin MY, Makarova KS, Wolf YI, Koonin EV. Expanded microbial genome coverage and improved protein family annotation in the COG database. Nucleic Acids Res. 2015;43:D261-9.

49. Hyatt D, Chen G, Locascio PF, Land ML, Larimer FW, Hauser L. Prodigal: prokaryotic gene recognition and translation initiation site identification. BMC Bioinformatics. 2010;11:119.

50. Marchler-Bauer A, Zheng C, Chitsaz F, Derbyshire MK, Geer LY, Geer RC, et al. CDD: conserved domains and protein three-dimensional structure. Nucleic Acids Res. 2013;41:348-52. 
51. Finn RD, Coggill P, Eberhardt RY, Eddy SR, Mistry J, Mitchell AL, et al. The Pfam protein families database: towards a more sustainable future. Nucleic Acids Res. 2016;44:D279-85.

52. Käll L, Krogh A, Sonnhammer ELL. Advantages of combined transmembrane topology and signal peptide prediction-the Phobius web server. Nucleic Acids Res. 2007:35:429-32.

53. Sullivan MJ, Petty NK, Beatson SA. Easyfig: a genome comparison visualizer. Bioinformatics. 2011;27:1009-10.

54. Stewart CA, Turner G, Vaughn M, Gaffney NI, Cockerill TM, Foster I, et al. Jetstream: A self-provisioned, scalable science and engineering cloud environment. Proc 2015 XSEDE Conf Sci Adv Enabled by Enhanc Cyberinfrastructure. 2015;:1-8. doi:https://doi.org/10.1145/2792745.2792774.

55. Towns J, Cockerill T, Dahan M, Foster I, Gaither K, Grimshaw A, et al. XSEDE: accelerating scientific discovery. Comput Sci Eng. 2014;16:62-74.

56. Levi K, Rynge M, Abeysinghe E, Edwards RA. Searching the sequence read archive using Jetstream and Wrangler. Proc Pract Exp Adv Res Comput. 2018:1-7. https://doi.org/10.1145/3219104.3229278.

57. Levi K. BAM scripts. Github 2017. https://github.com/kylelevi/BAM_scripts. Accessed 7 Jan 2017.

Ready to submit your research? Choose BMC and benefit from:

- fast, convenient online submission

- thorough peer review by experienced researchers in your field

- rapid publication on acceptance

- support for research data, including large and complex data types

- gold Open Access which fosters wider collaboration and increased citations

- maximum visibility for your research: over $100 \mathrm{M}$ website views per year

At $\mathrm{BMC}$, research is always in progress.

Learn more biomedcentral.com/submissions 